\title{
Are We on the Same Page? Patient and Provider Perceptions About Exercise in Cancer Care: A Focus Group Study
}

\author{
Agnes Smaradottir, MD; ; Angela L. Smith, MA; Andrew J. Borgert, $\mathrm{PhD}^{\mathrm{b}}$; and Kurt R. Oettel, MD
}

\begin{abstract}
Background: Physical activity (PA) during and after cancer treatment can help with symptom management and reduce the risk of cancer recurrence. However, it is unclear what constitutes an optimal exercise program. In addition, provider and patient barriers exist to the recommendation and adoption of exercise as part of a cancer treatment plan. The goal of this study was to determine how providers and patients feel about exercise during cancer treatment and explore what the barriers to implementing such a program might be. Patients and Methods: Focus groups and interviews were held with patients with malignancy, both metastatic and nonmetastatic, and oncology providers. In total, 20 patients participated in either a focus group or an individual interview and 9 providers contributed to the focus group. An equal number of patients $(n=10)$ were interviewed as attended a focus group. Audiotaped sessions were transcribed verbatim. Theme identification was independently coded by 4 coders and synthesized as a group. Results: Neither patient group recalled PA instruction from oncology providers during their cancer treatment. Most participants (95\%) felt exercise is important during cancer treatment, citing overall well-being benefits versus improved disease outcome. Most patients ( $80 \%$ ) preferred a home-based exercise program provided by the oncologist. Fatigue was the most cited barrier to regular exercise during treatment (50\%). All providers acknowledged benefits of PA to patients, but not universally for all. More than half of providers $(55 \%)$ preferred a referral system for exercise programs. Clinic visit time constraints and a perceived lack of expertise in the area of PA were common barriers to making exercise recommendations a routine part of the treatment plan. Conclusions: Patients with cancer and oncologists recognize the benefits of PA during treatment. Disagreement exists between to whom, how, and where exercise plans should be disseminated and implemented.
\end{abstract}

J Natl Compr Canc Netw 2017;15(5):588-594

\section{Background}

Each year, millions of Americans are faced with a cancer diagnosis, most often followed by complicated treatment plans involving multiple healthcare disciplines, such as surgery, radiation therapy, and chemotherapy. ${ }^{1}$ Often, a combination of these modalities is used, each with its own set of side effects that impact patients' motivation to be physically active. ${ }^{2}$

Emphasis on physical activity (PA) and its role in symptom management, reducing the risk of recurrence, and overall quality of life for patients with cancer has been a growing topic of investigation. Many studies have demonstrated a positive association between PA

From ${ }^{a}$ Gundersen Health System, and ${ }^{b}$ Gundersen Lutheran Medical Foundation, La Crosse, Wisconsin.

Submitted August 25, 2016; accepted for publication January 10, 2017.

The authors have disclosed that they have no financial interests,

arrangements, affiliations, or commercial interests with the manufacturers

of any products discussed in this article or their competitors. and improved outcomes for patients with cancer. ${ }^{2-4}$ But evidence also exists that the emotional and physical burden of treatment leads to a decline in PA and a failure to return to prediagnosis activity levels. ${ }^{5,6}$ Even though several cancer organizations have endorsed PA as an important part of cancer therapy, the design and implementation of an exercise program as part of a comprehensive cancer treatment plan remains unclear. ${ }^{7}$

Patients want advice and support about exercise while enduring the physiological and psychological side effects of treatment. ${ }^{8}$ Furthermore, they prefer that the exercise recommendation come from the oncology provider. ${ }^{9}$ Yet, very few patients in the United States report

Author contributions: Study conception and design, transcript coding, and drafting of manuscript: Smaradottir, Smith, Borgert, Oettel. Subject recruitment, focus groups, and interviews: Smith. Statistical analysis: Borgert.

Correspondence: Agnes Smaradottir, MD, Center for Cancer and Blood Disorders, Mail Stop EB2-001, Gundersen Health System, 1900 South Avenue, La Crosse, WI 54601. E-mail: asmarado@gundersenhealth.org 
receiving exercise recommendations as part of their cancer treatment plan. ${ }^{10}$ Limited resources, expert personnel, and knowledge of benefits are among the barriers to widespread implementation of patientcentered exercise programs in a cancer care center, which equates to lost potential benefits for patients. With this in mind, we sought to gain insight into barriers at both the provider and patient levels within our cancer center.

\section{Patients and Methods}

\section{Study Sample}

This study was reviewed and approved by the Gundersen Clinic, Ltd. Institutional Review Board. A query of our cancer center was performed to identify male and female patients with a cancer diagnosis, aged $\geq 45$ years, and receiving cancer treatment. Patients with breast cancer were excluded from this study because they have already been studied extensively. From the query, we identified 3 different groups of possible participants. Group 1 consisted of patients diagnosed with stage I-III cancer and who had finished adjuvant treatment in the past 6 months (nonmetastatic patients). Group 2 consisted of patients with metastatic disease currently receiving palliative chemotherapy (metastatic patients). Group 3 consisted of providers within our cancer center. From the list of eligible patients, invitations were sent to 46 stage I-III and 46 stage IV randomly selected patients for participation in a focus group to be held at Gundersen Health System. In total, 7 nonmetastatic and 3 metastatic subjects attended focus groups and 4 nonmetastatic and 6 metastatic patients agreed to individual interviews either via phone or during an appointment. Individual interviews were conducted with patients who wanted to participate in the study but were unable to attend the focus group sessions. No follow-up phone calls were placed to those patients who were invited but did not respond to the invitation. Providers were approached unannounced at a provider meeting. All providers in attendance at the meeting agreed to remain for the focus group and participated in the discussion.

\section{Procedure}

Upon agreement to participate in a focus group, subjects were mailed a personalized follow-up letter 1 week before the session detailing the logistics. Addi- tionally, the day before the scheduled focus group, a telephone call was made to confirm the participant's intent to attend.

The patient focus groups were conducted by the same moderator along with one observer and took place in the evening. The moderator for both patient focus groups was a trained educator from outside our institution with expertise in discussion facilitation. The observer/interviewer was a researcher with a background in education and similar expertise in facilitation skills. Informed consent was obtained, including consent for audio recording of the session. The same structured question bank, developed by the research team and tested prior to the focus group, was used for both groups. The questions consisted of an opening question, 2 introductory questions, 3 transition questions, 6 key questions, and a closing question (see supplemental eAppendix 1, available with this article at JNCCN.org). Individual interviews were conducted by the same researcher and audiotaped with prior consent from each participant.

Hematology and oncology providers were asked to participate in a focus group during a routine provider meeting. Consent was obtained to audiotape the session and was conducted by a moderator together with one observer. A structured questioning route of 1 opening question, 1 introductory question, 2 transition questions, 6 key questions, and a closing question was used in this focus group (supplemental eAppendix 2). Recordings from all focus groups and interviews were transcribed verbatim with deidentifying labels for participants.

After all focus groups, interviews, transcription, and coding were completed; chart review was performed to obtain demographic and clinical characteristics of participants. Electronic medical record review of 12 randomly selected patients for each provider was completed to determine the level of documented discussion about exercise initiated by providers. Data were also collected for provider demographics and personal exercise habits via one-onone questioning.

\section{Data Analysis}

Data from the interviews and focus sessions were individually coded by the research team of 2 physicians, an academic researcher, and a biostatistician. All coders separately read patient group transcripts and assigned individual statements to a category of choice based on 
Smaradottir et al

that statement. Similarly, the provider transcript was coded using this same constant comparative method. After individual coding was completed, the research team then met to discuss its findings, and key themes were identified from the categories each member identified, including definition of exercise, rehabilitation versus exercise, importance/benefits to cancer of exercise, barriers, priority, and motivation to engage in exercise. All subsequent analysis of transcripts used these themes and interviews continued until saturation was reached. All statistical comparisons used standard nonparametric tests, including chi-square or Fisher exact test for categorical comparisons and the Wilcoxon rank sum test for ordinal comparisons. A P value $<.05$ was considered significant for all comparisons. All calculations were performed with SAS 9.3 (SAS Institute Inc., Cary, NC).

\section{Results}

\section{Participants}

A total of 20 patients (11 nonmetastatic and 9 metastatic), with a mean age of 64 years $( \pm 7.8)$, participated in a focus group $(\mathrm{n}=10)$ or individual interview $(n=10)$. Metastatic and nonmetastatic participants were demographically and clinically similar (Table $1)$. The sample consisted of an equal representation of each sex. There were 11 different cancers associated with the participants (Figure 1). The

\begin{tabular}{|lccc|}
\hline Table 1. Patient Characteristics & & \\
Variable & $\begin{array}{c}\text { Nonmetastatic } \\
\text { Group } \\
(\mathbf{n}=11)\end{array}$ & $\begin{array}{c}\text { Metastatic } \\
\text { Group } \\
(\mathbf{n}=9)\end{array}$ & $\begin{array}{c}\boldsymbol{P} \\
\text { Value }\end{array}$ \\
\hline Age, mean (SD), y & $65( \pm 8.1)$ & $62( \pm 7.6)$ & .46 \\
\hline BMI, mean (SD) & $30.9( \pm 11.5)$ & $27.2( \pm 6.9)$ & .37 \\
\hline Male sex, N (\%) & $4(36)$ & $6(67)$ & .37 \\
\hline \begin{tabular}{l} 
Smoking status, N (\%) \\
\hline Current
\end{tabular} & $0(0)$ & $1(11)$ & .25 \\
\hline \begin{tabular}{l} 
Former \\
\hline Never
\end{tabular} & $5(45)$ & $6(67)$ & \\
\hline Treatment, N (\%) & $6(55)$ & $2(22)$ & \\
\hline Surgery & $7(64)$ & $3(33)$ & .36 \\
\hline $\begin{array}{l}\text { Radiation } \\
\text { Neoadjuvant }\end{array}$ & $2(36)$ & $1(11)$ & .32 \\
\hline Chemotherapy & $10(91)$ & $1(11)$ & .99 \\
\hline
\end{tabular}

Abbreviation: BMI, body mass index.

alndividual interview, $\mathrm{n}=4$.

'Individual interview, $\mathrm{n}=6$.

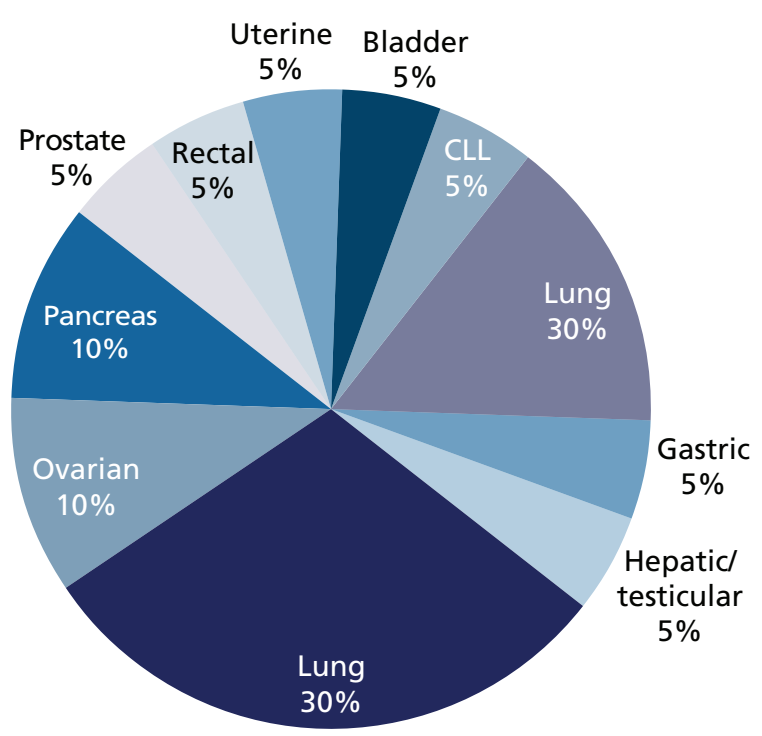

Figure 1. Participant cancer types.

Abbreviation: $\mathrm{CLL}$, chronic lymphocytic leukemia.

mean age of the providers $(n=9)$ was 45 years $( \pm 13)$, $56 \%$ were male, and the mean years of practice was $12( \pm 12.6)$. Most of the providers (89\%) themselves reported exercising on a regular basis. Chart review for the level of exercise recommendation to patients revealed that 2 of the 9 providers regularly initiated exercise discussions (Figure 2). No association between exercise discussion and clinical disease stage $(P=.68)$ or patient age $(P=.82)$ was found.

Exercise Recommendation During Treatment: Most participants did not recall any provider discussing and/or recommending an exercise program during their cancer treatment. Only one patient with metastatic disease recalled being instructed to exercise during treatment by an oncology provider. Two patients without metastatic disease recalled being instructed to exercise by their surgeon ("My surgeon did tell me he wanted me walking as much as I could, but after that I never heard anything about it").

Other participants reported actually being instructed to either reduce activity or not worry about PA when they inquired about exercise ("My doctor said, 'Don't worry about that right now, you have enough on your plate"'); this was common in both groups of patients (20\% and 30\%, respectively).

Most providers reported recommending PA to all of their patients. The main reason cited for such recommendations was large study results that showed benefit to patients with breast, colon, and prostate 


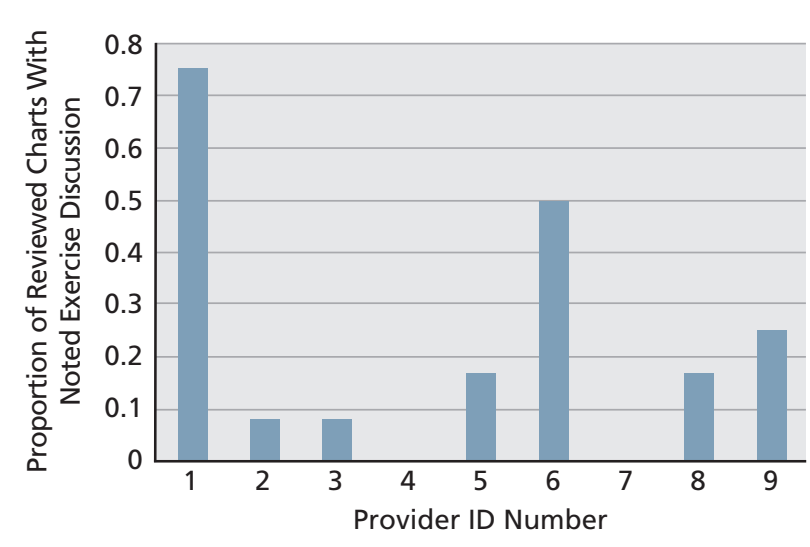

Figure 2. Documentation of exercise recommendation by provider.

cancers. However, some were skeptical about whether such recommendations would impact disease outcome ("I'm a skeptic. I don't know if the people who exercise are the ones who are going to do well anyway").

When discussing whether certain patient groups engage in PA, some providers reported feeling uncomfortable recommending increased activity to frail people who might have limited mobility and/or groups at risk of pathologic fractures. A question was also raised regarding how to motivate patients who have previously been very sedentary. In addition, concerns were expressed about asking patients to be more active when receiving chemotherapy and experiencing multiple side effects. But a counterpoint was raised that not discussing PA, in essence, gives patients permission to be inactive ("They think that you are not supposed to do anything while in treatment but lie in bed, so if you don't give them that verbal permission to get out there when they feel good, they will not").

Purpose of Exercise During Treatment: Most participants (95\%) felt regular activity/exercise was very important during their cancer treatment. It was equally important to both groups: those with nonmetastatic and metastatic cancers. Interestingly, most patients believed that regular daily activities constituted exercise. Most participants felt that the purpose of exercise was for overall well-being, to maintain function, and reduce stress ("To increase your muscle mass and recover more quickly"). Some felt empowered by a certain exercise routine ("Cancer has spurred me to exercise more, not that I'm trying to prolong my life. I want to make the most of every day that I have now and I feel better when
I exercise. I'm gonna live each day to the fullest"). None of the participants mentioned the possibility of affecting the outcome of their disease by engaging in a regular exercise regimen.

Exercise Program Design and Delivery: One of the key questions for the focus groups and interviews was "From whom and how would you like to receive exercise information?" Most participants (80\%) would like home-based exercise programs recommended by their oncologist. The most provided reason for wanting information and recommendations regarding exercise from their oncologist was their established relationship and that the oncologist would have extensive knowledge about what kind of treatment the participants had received and/or were receiving. In addition, they expressed feeling more comfortable receiving information about how much and what kind of exercise to engage in from someone who has firsthand knowledge about their disease status ("I rely a lot on my oncologist because they really do know").

More than half (55\%) of the providers would like to refer their patients to another healthcare professional (subspecialty) for exercise recommendations. Most of them felt they do not have enough insight to recommend specific exercise plans ("I'd like to see some kind of arrangement for some sort of referral... you're [the provider] not sure what to recommend").

Most providers voiced a perceived benefit of having an exercise physiologist on-site or a wellness facility within the cancer center. Significant discussion ensued about the benefit of the cancer rehabilitation program (Livestrong), which is available at the local YMCA, and the possibility of incorporating partnerships with an on-site wellness center where patients could exercise and receive guidance.

All providers felt that exercise is an important part of treatment plans for patients, but not for all patients universally. Only one provider regularly documented general exercise recommendations in patient charts, but others discussed it without documenting it. Patients often excluded from an exercise recommendation were those who were frail and those with extensive involvement of weight-bearing bone ("I worry about patients that have had bad compression fractures, especially with resistance training”).

Barriers to Exercise During Treatment: Both groups mentioned fatigue most often as a significant barrier to exercise (50\%). Other barriers included 
symptoms of disease, such as dyspnea, pain, and impaired mobility. Side effects of treatment, including nausea, vomiting, diarrhea, neuropathic pain, emotional state, and lack of motivation, also played a role in the ability to engage in regular PA.

Barriers to providers recommending exercise included time constraints of the clinic visit, lack of information about patient's physical fitness, and lack of knowledge about what kind of exercise program would be appropriate for each individual patient. Additionally, lack of transportation, cost of rehabilitation, and side effects of treatment, such as weakness and fatigue, were mentioned as factors that impact the decision to recommend exercise ("We think it's [exercise] important, but it's [the discussion] at the end of the appointment. It's not as important as the chemotherapy or the side effects from chemotherapy").

\section{Discussion}

Increased interest has been shown in the role of exercise as part of a comprehensive treatment plan for patients with cancer. Multiple studies suggest that patients with cancer prefer to receive exercise recommendations from their oncologist, but data also indicate that barriers are in place that prevent routine physician-initiated discussion about exercise as part of a treatment plan. ${ }^{11-14}$ In our study group, patients with and without metastatic disease consistently reported no recollection of physician-initiated conversations about exercise during their treatment. Jones and Courneya $\mathrm{a}^{15}$ found that most $(\approx 82 \%)$ cancer survivors prefer that their oncologist initiate exercise discussions; still less than a third (28\%) of survivors reported having had these discussions initiated by their oncologist. Similarly, even though nearly all of our patients rated the importance of exercise as very high, conversations occurring between oncologists and patients were nonexistent. From a physician perspective, there seems to be a lack of engagement. The provider group resoundingly agreed that exercise is beneficial to patients with cancer. These results parallel those of previous studies reporting that $73 \%$ and $62 \%$ of oncologists agree that exercise is beneficial. ${ }^{13,14}$ Yet only 2 providers in our group documented exercise recommendation in the electronic medical record $>50 \%$ of the time; for the remainder, this rate was $\leq 25 \%$. Our results are consistent with those of the Canadian provider group reporting a $28 \%$ recommendation rate. ${ }^{9}$ The provider focus group responses suggested that reduced engagement was related to a perceived lack of expertise in the field of exercise, and it was even suggested that perhaps physicians were not the correct group to dispense this type of information. These results pose a conundrum. The evidence clearly supports exercise as beneficial to patients with cancer in terms of recovery from treatment, reduced risk of recurrence, and improved survival; both physicians and patients acknowledge this, so it is unclear why there is a disconnect.

Cancer treatment modalities have become more complex over the past few decades. A patient undergoing treatment requires management of multiple ongoing needs. Multimodality treatment involves multiple subspecialties, and therefore requires patients to have contact with more providers during treatment than in the past - upwards of a dozen providers, with the potential for additional appointments for patients with more severe side effects. We found that endless clinic visits played a role in our patients' preference for receiving exercise recommendations in the form of a home-based program with specific instructions (written and verbal) for what to do, how long, and at what intensity. Furthermore, the general consensus was that such a program should be individualized for the specific patient's disease status. This is consistent with other findings concluding that current recommendations are generic and that future exercise programs should take the target population into account in order to foster adherence and longstanding behavioral change. ${ }^{16,17}$ Traditionally, the physician's role has been to address the issues within their area of expertise and consult or refer out for those outside their realm of expertise. There is now a dilemma in prioritizing where exercise fits on the treatment spectrum and balancing the needs of the patient with the comfort level of the physician.

Our results indicate that exercise is perceived as important to cancer survivors, both from the patient and physician perspective. We have also concluded that patients want to hear this information from physicians; however, physicians are reluctant to consistently include PA discussions for a number of reasons. These findings highlight the value of examining both patient and provider attitudes and behavioral intentions. The strengths of this study are that both physicians and patients from the same institution were interviewed, providing an understanding about 
how the group as a whole may influence effective implementation of good practice. The limitations of this study include sample size and the mixed format of data gathering (focus groups and individual interviews).

The question remains how we bridge the gap that exists between patient and provider preferences when it comes to PA as part of cancer treatment. First, physician education should be paramount. If any progress in this field is to be made, physicians must feel comfortable providing PA information and feel that it is of value to patients. To do this, there should be some formal collaboration with physical therapy, exercise physiology, or other subspecialty in providing guidelines for exercise. Integrating a PA specialist into the oncology clinic visit would fundamentally facilitate effective exercise planning and set the stage for longer-term behavior change. Physicians would gain a good sense of scaled exercise recommendations accounting for the multiple comorbidities with which patients present through shared care with a PA specialty. This emphasis should be on a "patient-centered care" approach, making the recommendations specific for each patient and his/her disease. Physicians, fellows, and residents who collaborate with a PA specialist through a shared-care clinic visit will be gaining valuable education about how to discuss exercise recommendations with their patients.

The Institute of Medicine and American Cancer Society include PA in their health promotion guidance recommendations. ${ }^{18}$ In addition, the survivorship curriculum developed by ASCO details physician engagement about health promotion topics, such as smoking cessation, weight management, and PA, as paramount to providing quality cancer survivorship care. Furthermore, a shared-care model is encouraged to achieve success in these areas. ${ }^{19} \mathrm{~A}$ collaborative shared-care model has the potential to benefit all stakeholders: patients, providers, institutions, and third-party payers. Based on our institution's billing practice surrounding collaborative care, patients would not incur any additional expenses associated with a PA specialist being a part of a shared-care visit in the cancer center. Not all institutions have an infrastructure that would support these services and is an area in need of attention.

The Breast Cancer Survivorship Program within our institution includes a survivorship plan that is shared with primary care providers. These survivorship plans are initiated at the time of diagnosis versus at the conclusion of treatment. Data support that patients are highly motivated to make lifestyle changes at the time of initial diagnosis and may serve as an ideal "teachable moment" for health promotion interventions. ${ }^{20}$ Incorporating a recommendation for PA based on the integrated appointment with a PA specialist during the initial treatment phase, and along the course of survivorship, would be an excellent model for sharing information with the primary care provider. We suggest that expanding survivorship programs to other cancers inclusive of a collaborative care model promoting PA interventions could be an effective solution to breaking down the barriers that exist to routinely integrate this service into cancer care delivery.

\section{Conclusions}

Our study finds that exercise recommendations are important from both a patient and physician perspective, but there remains a disconnect between how physicians can best advocate for PA and how to best provide an exercise plan to individual patients. We propose that integrating PA specialists into oncology visits may be bridge the gap that exists.

\section{Acknowledgments}

The authors would like to thank Jennifer Voigt for her assistance as the focus group moderator, and Anna M. Curtis for proofreading the manuscript.

\section{References}

1. American Cancer Society. Cancer Facts \& Figures 2015. Available at: https://old.cancer.org/acs/groups/content/@editorial/documents/ document/acspc-044552.pdf. Accessed March 3, 2017.

2. Courneya, KS. Exercise interventions during cancer treatment: biopsychosocial outcomes. Exerc Sports Sci Rev 2001;29:60-64.

3. Sui $\mathrm{X}$, Lee DC, Matthews $\mathrm{C}$, et al. The influence of cardiorespiratory fitness on lung cancer mortality. Med Sci Sports Exerc 2010;42:872-878.

4. Alfano CM, Klesges RC, Murray DM, et al. Physical activity in relation to all-site and lung cancer incidence and mortality in current and former smokers. Cancer Epidemiol Biomarkers Prev 2004;13:2233-2241.

5. Huesbo AM, Karlsen B, Allan H, et al. Factors perceived to influence exercise adherence in women with breast cancer participating in an exercise programme during adjuvant chemotherapy: a focus group. J Clin Nurs 2015;24:500-510. 


\section{Smaradottir et al}

6. Rogers LQ, Courneya KA, Shah P, et al. Exercise stage of change, barriers, expectations, values and preferences among breast cancer patients during treatment: a pilot study. Eur J Cancer Care (Engl) 2007;16:55-66.

7. Balogh EP, Ganz PA, Murphy SB, et al. Patient-centered cancer treatment planning: improving the quality of oncology care. Summary of an Institute of Medicine workshop. Oncologist 2011;16:1800-1805.

8. Murnane A, Geary B, Milne D. The exercise programming preferences and activity levels of cancer patients undergoing radiotherapy treatment. Support Care Cancer 2012;20:957-962.

9. Jones LW, Courneya KS. Exercise discussions during cancer treatment consultations. Cancer Pract 2002;10:66-74.

10. Denmark-Wahnefried W, Peterson B, McBride C, et al. Current health behaviors and readiness to pursue life-style changes among men and women diagnosed with early stage prostate and breast carcinomas. Cancer 2000;88:674-684.

11. Jones LW, Courneya KS. Exercise counseling and programming preferences of cancer survivors. Cancer Pract 2002;10:208-215.

12. McGowan EL, Speed-Andrews AE, Blanchard CM, et al. Physical activity preferences among a population-based sample of colorectal cancer survivors. Oncol Nurs Forum 2013;40:44-52.
13. Jones LW, Courneya KS, Peddle C, Mackey JR. Oncologists' opinion towards recommending exercise to patients with cancer: a Canadian national survey. Support Care Cancer 2005;13:929-937.

14. Park JH, Oh M, Yoon YJ, et al. Characteristics of attitude and recommendation of oncologists toward exercise in South Korea: a cross sectional survey study. BMC Cancer 2015;15:249.

15. Jones LW, Courneya KS. Exercise discussions during cancer treatment consultations. Cancer Pract 2002;10:66-74.

16. Szymlek-Gay EA, Richards R, Egan R. Physical activity among cancer survivors: a literature review. NZ Med J 2011;124:77-89.

17. Buffart LM, Galvao DA, Brug J, et al. Evidence-based physical activity guidelines for cancer survivors: current guidelines, knowledge gaps an future directions. Cancer Treat Rev 2014;40:327-340.

18. Demark-Wahnefried W, Rogers LQ, Alfano CM, et al. Practical clinical interventions for diet, physical activity and weight control in cancer survivors. CA Cancer J Clin 2015;65:167-189.

19. Shapiro CL, Jacobsen PB, Henderson T, et al. ASCO core curriculum for cancer survivorship education. J Oncol Pract 2016;12:145, e108-117.

20. Demark-Wahnefried W, Aziz NM, Rowland JH, Pinto BM. Riding the crest of the teachable moment: promoting long-term health after the diagnosis of cancer. J Clin Oncol 2005;23:5814-5830.

See JNCCN.org for supplemental online content. 